\title{
Underwater Sound Speed Inversion by Joint Artificial Neural Network and Ray Theory
}

\author{
Wei Huang \\ School of Electronic Information \\ Wuhan Universy \\ Wuhan, Hubei Province, China \\ whuwsnhw@whu.edu.cn
}

\author{
Deshi $\mathrm{Li}^{*}$ \\ School of Electronic Information \\ Wuhan Universy \\ Wuhan, Hubei Province, China \\ dsli@whu.edu.cn
}

\author{
Peng Jiang \\ National Engineering Center for \\ Satellite Positioning System \\ Wuhan Universy \\ Wuhan, Hubei Province, China \\ jiangp@whu.edu.cn
}

\begin{abstract}
Sound speed profiles (SSPs) have a great impact on the accuracy of underwater localization and sonar ranging. In traditional SSP inversion, the sound intensity distribution used in normal mode theory-based matching field processing (MFP) or the multipath signal propagation time adopted in ray theory-based MFP is susceptible to boundary parameter mismatch issues, which reduces the inversion accuracy. Moreover, heuristic algorithms introduced in the MFP require many individuals and iterations to search for the optimal feature representation coefficients after the empirical orthogonal function (EOF) decomposition, which causes extra computational time. In this paper, we propose a two-way interactive signal propagation time measurement method based on an autonomous underwater vehicle (AUV) and a horizontal linear array (HLA), and we apply the propagation time of direct arrival signals for shallow-water SSP inversion to avoid the boundary parameter mismatch. We propose a joint artificial neural network (ANN) and ray theory SSP inversion model to reduce the computational time at the working phase by fitting the nonlinear relationship from the signal propagation time to the SSP, and once the relationship is established, the goal of reducing the computational time can be achieved. To make the ANN better learn the SSP distribution in a target region and ensure a good inversion accuracy, we give an empirical data selection strategy. Then we propose a virtual SSP generation algorithm to help ANN training in the case of under-fitting problems caused by insufficient training data. Simulation results show that our approach can provide a reliable and instantaneous monitoring of shallow-water SSP distribution.
\end{abstract}

\section{KEYWORDS}

artificial neural network (ANN), direct arrival signals, ray theory, sound speed profile (SSP), sparse feature points (SFPs)

ACM Reference Format:

Wei Huang, Deshi Li, and Peng Jiang. 2018. Underwater Sound Speed Inversion by Joint Artificial Neural Network and Ray Theory. In The 13th ACM International Conference on Underwater Networks \& Systems (WUWNet'18),

${ }^{*}$ Corresponding Author

Permission to make digital or hard copies of part or all of this work for personal or classroom use is granted without fee provided that copies are not made or distributed for profit or commercial advantage and that copies bear this notice and the full citation on the first page. Copyrights for third-party components of this work must be honored.

For all other uses, contact the owner/author(s).

WUWNet'18, December 3-5, 2018, Shenzhen, China

(C) 2018 Copyright held by the owner/author(s).

ACM ISBN 978-1-4503-6193-4/18/12.

https://doi.org/10.1145/3291940.3291972
December 3-5, 2018, Shenzhen, China. ACM, New York, NY, USA, 8 pages. https://doi.org/10.1145/3291940.3291972

\section{INTRODUCTION}

Sound speed profiles (SSPs) have a great impact on the accuracy of underwater localization [7, 16] and sonar ranging [2]. An SSP describes the distribution of underwater sound speed in a certain area at different vertical depths. Although given as a function of depth, the speed of sound actually depends on the temperature, static pressure, and salinity at a certain depth [4]. The SSP can be directly measured by a sound velocity profiler (SVP), and it can be calculated through empirical formula, which makes use of the temperature, salinity, and depth information measured by a conductivity-temperature-depth (CTD) system. However, the use of the SVP and CTD system is labor intensive and energy consuming due to the need for manual on-site operation. Besides the direct measurement method, the SSP can also be obtained by inversion of sound field information. As the inversion method facilitates longterm observation of ocean parameters and reduces labor costs as well as energy consumption, there has been growing interest in the engineering realization of underwater SSP inversion.

There are two major theoretical and conceptual frameworks for SSP inversion: the acoustic ray theory [9] and normal mode theory $[10,12]$. Munk and Wunsh [9] put forward the underwater acoustic ray theory and explained basic principles of ocean acoustic tomography (OAT), which laid a theoretical foundation for the inversion of SSP. To further take the boundary effects into consideration and increase the accuracy of SSP inversion, Munk and Wunsh [10] and Shang [12] proposed the normal mode theory. Through either of these two theories, the one-way mapping from ocean environmental information to sound field information can be established, but till now, there has been no theoretical support for the reverse process. Therefore, the SSP is difficult to be directly inversed from sound field information. Tolstoy et al. [15] combined a matched field processing (MFP) technique with empirical orthogonal function (EOF) decomposition for SSP inversion. In this approach, the reverse mapping from sound field information to ocean environment information is avoided, making the MFP technique a mainstream solution for SSP inversion.

Recently, some SSP inversion approaches using the MFP technique have been proposed, which are theoretically based on normal mode theory $[1,5,6]$ or ray theory $[13,14,17-20]$. Although the sound intensity distribution of the sound shadow zone, convergence zone, and caustics zone can be accurately described by the normal mode theory, it is vulnerable to the boundary parameter mismatch, 
complex in computation and unintuitive for understanding. Conversely, acoustic ray theory has the advantages of intuitiveness and low computational complexity. In [13, 14, 17-20], multipath signal propagation time was used as the matching objective parameter for SSP inversion. By taking advantage of the multipath effect, the medium coverage is improved. However, a wedge-shaped sea floor will cause deflection of the horizontal sound ray $[17,18]$. As a result, it is difficult to restore the signal propagation time accurately when the boundary parameter does not match sufficiently in the process of matching field calculation, which reduces the accuracy of SSP inversion.

There is a further problem with MFP-based SSP inversion; the time overhead at the working phase cannot be ignored from the perspective of saving energy. One issue is that part of the time consumption comes from calculating the matching parameters through theoretical models. In $[17,18]$, a perturbation method was proposed to convert the nonlinear integrals into a linear system of equations, by which the computational complexity could be reduced, whereas the inversion accuracy is sacrificed. A second issue is that the time overhead is affected by the efficiency of the searching algorithm as well. However, it is usually time consuming to search for the optimal EOF coefficients, which increases the energy consumption and reduces the life cycle of underwater nodes. In [5], the coefficient search space was reduced first, and then a traversal principle was used to find the optimal solution, while in [6] a parallel grid search algorithm was proposed to reduce time consumption for searching. However, the searching accuracy depends on the scanning step, which is why the the time overhead increases as the accuracy of SSP inversion improves. Heuristic optimization algorithms were introduced in $[13,14,17-20]$ to speed up the searching process of the optimal EOF coefficients, such as the simulated annealing (SA) algorithm in [19], genetic algorithm (GA) in [13, 14], and particle swarm optimization (PSO) algorithm in $[17,18,20]$. However, to get the optimal result with a high probability, multiple iterations with multiple individuals are necessary in these heuristic algorithms. Consequently, the time overhead can not be reduced to a desired level.

To quickly obtain a reliable SSP reference for shallow-water applications, several problems should be resolved. First, the sound intensity distribution and the multipath signal propagation time respectively adopted by normal mode theory based MFP and ray theory based MFP are susceptible to the boundary parameter mismatch. Second, heuristic algorithms used in MFP require many individuals and iterations to search for the optimal feature representation coefficients after the EOF decomposition, which is time-consuming. To avoid the boundary parameter mismatch problem, the propagation time of direct arrival signals is used for SSP inversion in this paper. However, the traditional measurement method for measuring the sound intensity distribution or the multipath signal propagation time cannot be directly applied to the measurement of the direct arrival signal propagation time because of stability and coverage issues. In [13, 14, 17, 18, 20], a vertical linear array (VLA) was used as the receiver, but the attitude of the VLA is unstable due to the influence of water current. To overcome this problem, a source node and a horizontal linear array (HLA) that were both moored on the seabed were used in [5, 6]. Nonetheless, the source node needs to be replaced as the propagation path of the direct arrival signal cannot cover the whole column this way. To overcome the stability and coverage problems, we take advantage of the HLA and introduce a shallowly suspended autonomous underwater vehicle (AUV) as the source node. Thus, the transceiver nodes can ensure that the sound wave will record the whole column of information during the propagation process. Because the AUV is able to actively adjust its position and attitude, the stability of our measurement system can be guaranteed. Secondly, the MFP-based SSP inversion method faces with high time consumption due to massive match searching calculation. As artificial neural networks (ANNs) can handle nonlinear calculations [3], we introduce an ANN to learn the mapping relationship from the propagation time information to the distribution of sound speed. Once the mapping relationship is established, the goal of reducing time consumption at the working phase can be achieved. Moreover, ray theory is needed in our approach to calculate the theoretical signal propagation time information for each set of empirical SSP data, so the required input information of the mapping relationship to be fitted by the ANN is provided.

Over-fitting and under-fitting are two main problems that appear during the training phase of an ANN. The over-fitting problem means that the generalization ability of the ANN is deteriorated, and the potential for over-fitting depends on both the training data selection and model structure of the ANN such as the number of layers and the number of hidden layer neurons. The under-fitting problem indicates that the ANN cannot adequately capture the underlying structure of the input data, and this usually occurs when the training data or training times are insufficient, or the features of training data are too scattered to be learned. To ensure good performance of the ANN, the training data need to be carefully selected and the structure of the ANN should be well designed. In this paper, we propose a joint ANN and acoustic ray theory method for shallow-water SSP inversion, which makes use of the propagation time of direct arrival signals as the sound field information. The contributions of our work include:

(1) We present an AUV- and HLA-based two-way interactive method for measuring the propagation time of direct arrival signals in shallow water to reduce the influence of boundary parameter mismatch. To deal with the stability and signal coverage issues, an AUV is suspended near the ocean surface as a source node, and a receiving HLA is moored on the seabed. A two-way interaction is introduced to solve the problem of time asynchrony.

(2) We propose a joint ANN and ray theory SSP inversion model. The ANN is adopted to fit the complex nonlinear relationship from the signal propagation time to the ocean SSP. Compared with traditional MFP based SSP inversion methods, our approach can significantly reduce the calculation time at the working phase while ensuring the accuracy of the SSP inversion.

(3) We propose an empirical SSP data selection strategy to provide accurate training data for a certain task and put forward a virtual SSP data generation algorithm to help ANN training. By training the ANN through a combination of the empirical and virtual SSP data, the under-fitting problem of the ANN caused by insufficient training data can be solved. As the virtual SSP data are generated according to the distribution 
of the empirical SSP data, the consistency of the SSP distribution to be learned by the ANN and that of the empirical SSP can be guaranteed.

To verify the feasibility and effectiveness of our approach, we conducted a simulation experiment to quantify the inversion accuracy and time consumption.

The rest of the paper is organized as follows. The two-way interactive measuring method of the propagation time of direct arrival signals is proposed in Sec. 2. The system model and parameters are proposed and described in Sec. 3. The simulation results are discussed in Sec. 4, and conclusions are presented in Sec. 5.

\section{MEASUREMENT OF SOUND PROPAGATION TIME}

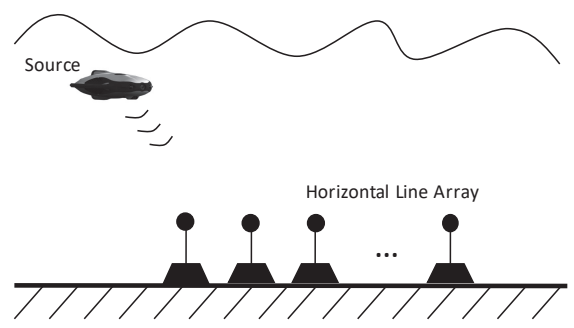

Figure 1: Propagation time measuring.

Unlike multipath signals, the direct arrival signal is not affected by boundary reflection during the propagation process. To avoid the boundary parameter mismatch, the propagation time of direct arrival signals is applied as the sound field information for SSP inversion in this paper. However, the transceiver nodes should be separately deployed near the ocean surface and floor to ensure that the sound wave can record all environmental column information during the propagation process. In addition, to reduce the propagation time measurement error when there is only a single pair of sender and receiver, it is necessary to measure multiple sets of propagation time data with an array at either the transmitting end or the receiving end. Considering that the attitude of a VLA is unstable due to the water current, an HLA moored on the sea floor becomes a better choice $[5,6]$ to reduce measurement errors caused by attitude-unstable receivers. Thus, we propose an AUVand HLA-based direct arrival signal propagation time measurement method for SSP inversion, as shown in Fig. 1. To deal with the signal coverage problem, the HLA is moored on the seabed, and the AUV is shallowly suspended in the same profile with the HLA. Because the position of the HLA is fixed, the stability of receivers can be ensured. To reduce the influence of water flow on the transmitting node and ensure that the transmitting node and the HLA are in the same profile, an AUV is introduced as the sender, which has the ability to actively adjust its position and attitude. It should be noted that it is better to minimize the horizontal distance between the AUV and HLA. For example, the AUV can be vertically above the HLA edge node, so that the difference in time of receiving signals from different HLA elements can be expanded, thereby enhancing the ability to distinguish sound speed distribution. Moreover, the communication power requirements can be reduced. On this basis, the propagation time of direct arrival signals, which is expressed as a time vector $\vec{t}=\left[t_{1}, t_{2}, \ldots, t_{N}\right]$, can be measured by communication between the transceiver nodes, where the vector item $t_{n}, n=1,2, \ldots, N$ represents the signal propagation time between the AUV and the $n$th HLA node.

To reduce the measuring error of signal propagation time caused by time asynchrony between transceiver nodes, a two-way interaction process is introduced as in Fig. 2. An AUV first sends an initialization (INIT) message, and after a back-off period, each node of the HLA separately replies with an acknowledgment (ACK) message. Assume that the HLA is made up of $N$ nodes. $H n$ represents the $n$th HLA node, and the AUV is marked as $A$. The propagation time of the direct arrival signal between the $n$th pair of transceiver nodes is expressed as:

$$
t_{n}=\frac{1}{2}\left(t_{r}^{H n}-t_{s}^{A}+t_{r n}^{A}-t_{s}^{H n}\right), n=1,2, \ldots, N
$$

where $t_{s}^{A}$ is the local sending time of the INIT message at $A, t_{r}^{H n}$ is the corresponding local receiving time of the INIT message at the $n$th HLA node, $t_{s}^{H n}$ is the local time of the $n$th HLA node when the ACK message is replied, and $t_{r n}^{A}$ is the corresponding local receiving time of the ACK message at $A$. Assume that the clock of the AUV is ahead of the clock of the $n$th HLA node, and the time difference is $\Delta t^{A-H n}$. Then the Eqn.(1) can be modified as:

$t_{n}=\frac{1}{2}\left(\left(\tau^{H n-A}+\Delta t^{A-H n}\right)+\left(\tau^{A-H n}-\Delta t^{A-H n}\right)\right), n=1,2, \ldots, N$,

where $\tau^{H n-A}$ is the propagation time of the INIT message sent by $A$, and $\tau^{A-H n}$ is the propagation time of the ACK message sent by the $n$th HLA node. Referring to Eqn.(2), the time synchronization error $\Delta t^{A-H n}$ is finally eliminated by subtraction in the formula, so the measuring error of signal propagation time caused by time asynchrony between transceiver nodes is reduced. As a result, the final signal propagation time is an average value of the propagation time of the INIT message and the ACK message, which is expressed as:

$$
t_{n}=\frac{1}{2}\left(\tau^{H n-A}+\tau^{A-H n}\right), n=1,2, \ldots, N
$$

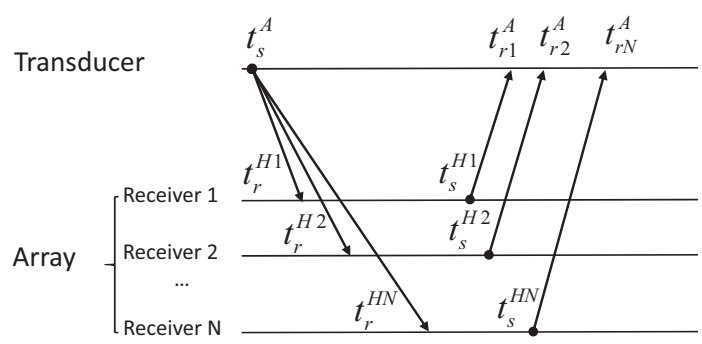

Figure 2: Two-way interaction process. 


\section{JOINT ANN AND RAY THEORY MODEL FOR SSP INVERSION}

\subsection{Structure of Joint ANN and Ray Theory Model}

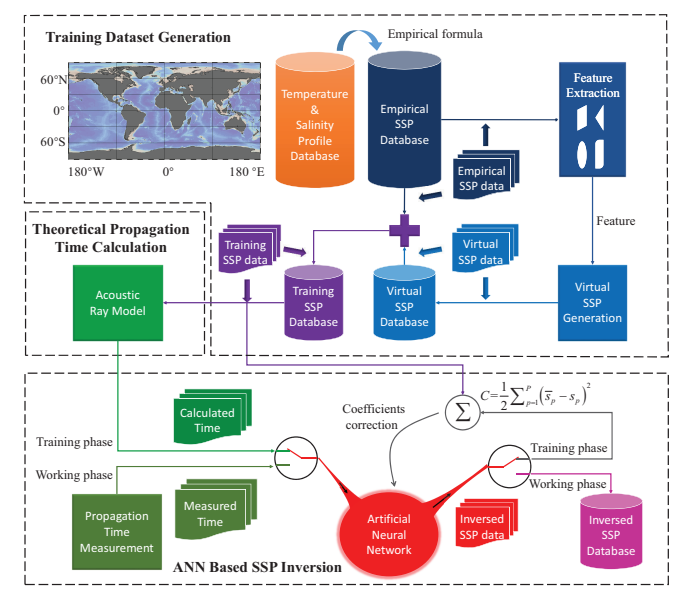

Figure 3: Structure of joint ANN and ray theory model.

Low energy consumption is important for extending the life cycle of an SSP inversion system. The energy consumption during the SSP inversion process mainly comes from the communication interaction process when measuring the signal propagation time and the dynamic power consumption of hardware processors when processing data. One way to save the system energy is to reduce the computational time under a certain hardware resource occupancy at the working phase; thus, we propose a joint ANN and ray theory model for SSP inversion to reduce the calculation time at the working phase while ensuring high accuracy.

The core idea of our approach is to establish the nonlinear mapping relationship from the propagation time of direct arrival signals to the corresponding distribution of sound speed achieved by the ANN. The structure of the joint ANN and ray theory model is shown in Fig. 3. It is composed of three parts: a training dataset generation module, theoretical propagation time calculation module, and ANN-based SSP inversion module.

To obtain a good SSP inversion accuracy at the working phase, the ANN needs to be well trained during the training phase. To ensure that the training data accurately reflect the SSP in a target area, we propose an empirical dataset selection strategy. However, because it is labor intensive and energy consuming to measure SSPs by a SVP or CTD system, there is not enough empirical data for ANN training in most objective regions for a same short time period of each year. To avoid the under-fitting problem caused by insufficient training data, we propose a virtual SSP data generation algorithm, and the virtual SSP dataset generated is combined with the empirical SSP dataset for ANN training. After the training dataset generation, the acoustic ray theory is introduced to calculate the theoretical propagation time for a certain SSP. In this way, the indispensable input information of the mapping relationship to be fitted by the ANN is provided. At the working phase, the SSP inversion result is obtained by inputting the measured time information into the trained neural network.

The SSP inversion workflow is summarized as:

(1) Appropriate empirical SSP data selection and virtual SSP data generation;

(2) Theoretical propagation time calculation based on acoustic ray theory;

(3) ANN training offline;

(4) Real propagation time measurement in the target sea area;

(5) SSP inversion via the trained ANN.

\subsection{Training Dataset Generation}

3.2.1 Empirical SSP Selection Strategy. The convergence of ANN is related to the training dataset. For regression analysis, an inappropriate training dataset will increase the inversion error, even leading to erroneous results. In fact, the speed of sound is regularly distributed and mainly determined by temperature factors in shallow water, which makes it indirectly related to regions and seasons. In this paper, 340 sets of SSP data from the world ocean
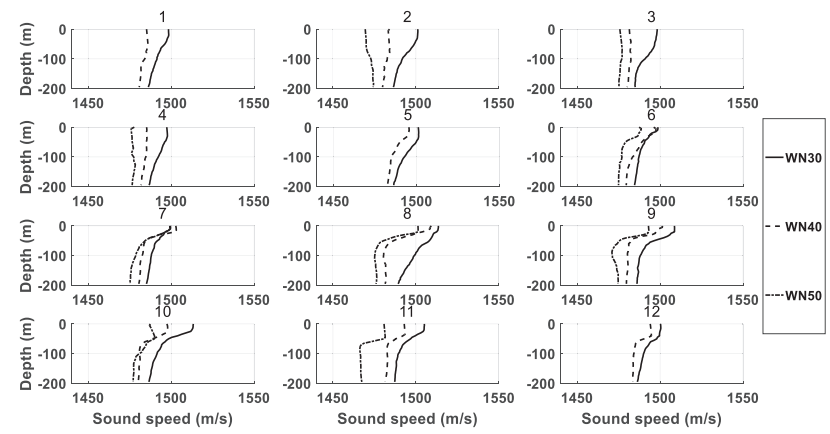

Figure 4: Typical shallow-water SSPs in different months along the eastern Pacific coast between 30-60 degrees north latitude with depth of $200 \mathrm{~m}$.

database 2013 (WOD13) are analyzed, collected in shallow water along the eastern Pacific coast between 30-60 degrees north latitude. Some typical SSPs are presented in Fig. 4. Shapes of the SSP data vary in different months in the same region, and the depth of the sound speed turning point is floating in a certain month at different latitudes. However, for a same time period of each year, the SSPs measured in a certain region has a similar distribution. As the sampled region and time of the empirical data get closer to the region and historical contemporaneous time of the task, the similarity of these SSPs increases. Therefore, to better describe the possible SSP distribution in a target area, we propose an empirical SSP data selection strategy to choose the empirical SSP data that has a closer sampled region with historical contemporaneous time according to the task.

3.2.2 Virtual SSPS Generation. To solve the under-fitting problem caused by insufficient training data, we propose a virtual SSP data generation algorithm, and the ANN is trained by a combination of the empirical and virtual SSP data. Assuming that the empirical SSP data selected according to the strategy proposed in this paper 
are able to accurately describe the SSP distribution in a target region, the virtual SSP distribution should be consistent with that of the empirical SSP data to make sure that the virtual SSP data will not lead to bad ANN learning outcomes. Therefore, we propose a sparse feature point (SFP) extraction method to obtain the distribution of the empirical SSP data, and the virtual SSP data are generated according to the SFP extraction result. By these methods, the consistency and under-fitting problems can be solved.

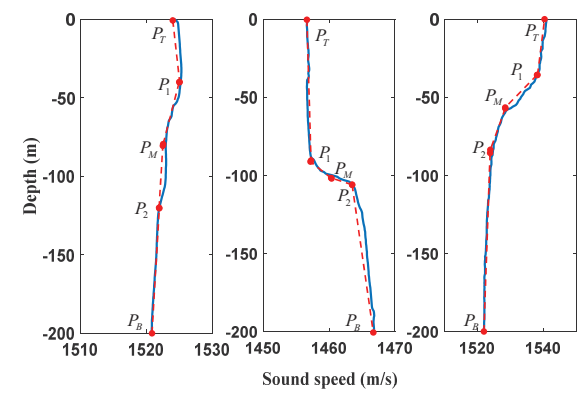

Figure 5: The SFP extraction of typical SSPs in shallow water.

SSPs Classification and SFPs Extraction. The empirical SSP distribution is the reference basic of the virtual SSP data to be generated; however, the empirical SSP distribution varies in different months and regions. To design a general feature extraction method for SSPs, statistical analysis and summarization of a large number of empirical SSPs are required. Referring to Fig. 4, there are mainly three types of SSPs in shallow water, which are described as shown in Fig. 5. The three types of SSPs are described as the slanted type, the positive gradient "S" type and the negative gradient "S" type. No matter which type the SSP belongs to, a five-point-based fold line can be adopted to approximately express the entire curve, and the five points are called SFPs. The pseudo code of the SFP extraction process is presented in Table 1 , where $P_{T}, P_{B}, P_{M}, P_{1}$, and $P_{2}$ respectively represent the surface point, and the bottom point, the middle point, and the turning point 1 and 2. $P_{1}$ and $P_{2}$ are chosen according to an approximation principle of minimum mean squared error (MSE) given in Definition 3.1.

Definition 3.1. If $P_{1}$ (or $P_{2}$ ) is a turning point, $P_{1}$ (or $P_{2}$ ) must be a point between point $P_{M}$ and $P_{T}$ (or $P_{B}$ ) on the SSP curve. Meanwhile, among all candidate positions of $P_{1}$ (or $P_{2}$ ), $P_{1}$ (or $P_{2}$ ) must be at the position where the MSE between the fold line $\overline{P_{M} P_{1} P_{T}}$ (or $\overline{P_{M} P_{2} P_{B}}$ ) and the sub-curve $P_{M} \widehat{P}_{1} P_{T}\left(\right.$ or $\left.P_{M} \widehat{P}_{2} P_{B}\right)$ is minimum.

Virtual SSP Generation Algorithm. We take the region of the eastern Pacific coast between 38-39 degrees north latitude in June as an example to explain the virtual SSP generation algorithm, and the SFP extraction results are given in Fig. 6. Referring to Fig. 6, some rectangles are employed to represent the value space of the SFPs. The virtual top point $P_{T}^{V}$, bottom point $P_{B}^{V}$, and middle point $P_{M}^{V}$ are generated following a uniform distribution according to the upper and lower bounds of the SFP extraction results. Noting
Table 1: The SFP extraction of SSPs in shallow water

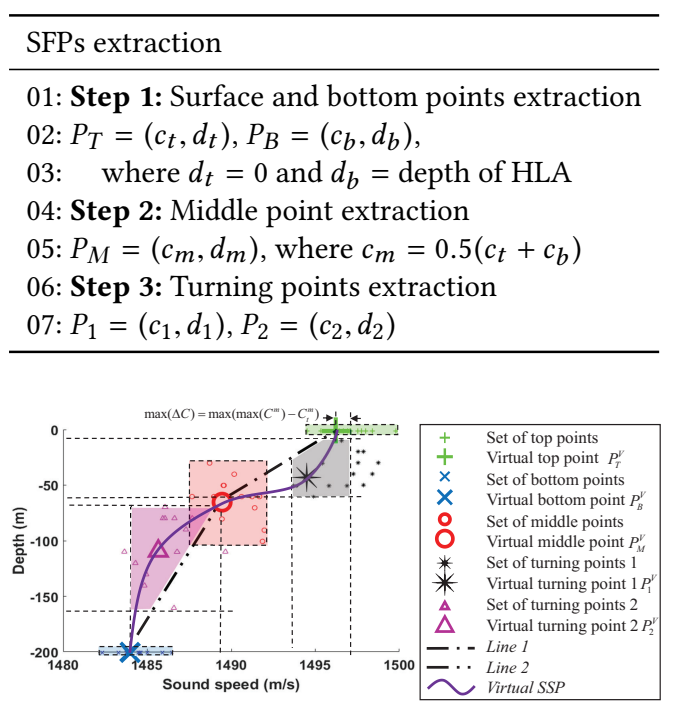

Figure 6: Virtual SSP generation for shallow water

that the SSP is usually monotonous and the sub-curves ${ }_{P_{T}^{V}}^{V}{ }_{M}^{V}$ and $P_{B}^{V P} P_{M}^{V}$ are single convex in the "S" type, we introduce two auxiliary lines $\overline{P_{T}^{V} P_{M}^{V}}$ and $\overline{P_{B}^{V} P_{M}^{V}}$ to reduce the solution space of the virtual turning points $P_{1}^{V}$ and $P_{2}^{V}$. This method is also suitable for the slanted distribution, as it can be considered as a compression transformation of the "S" type. Due to the influence of temperature, there will be a weak positive gradient phenomenon in the shallow water over some months. To imitate this characteristic and control the amplitude of the positive gradient, a parameter $\Delta C$ is proposed to describe the difference between the maximum sound speed and the surface sound speed value of each set of SSP. The pseudo code of the virtual SSP generation algorithm for negative gradient "S" type SSPs as an example is given in Table 2, where $C$ and $D$ are respectively the SFP sets of the sound speed and depth of the empirical SSP data, whereas $c$ and $d$ represent the sound speed and depth of the virtual generated points.

\subsection{Theoretical Propagation Time Calculation}

Acoustic ray theory could establish the mapping relationship from the SSP distribution to the signal propagation time information, which is the indispensable input information of the mapping relationship to be fitted by the ANN. For a given SSP, the horizontal propagation distance at the $n$th HLA node is calculated by:

$$
x^{n}=\sum_{i=0}^{m} \Delta x_{i}=\frac{c_{0}}{\cos \theta_{0}^{n}} \sum_{i=0}^{m}\left|\frac{\sin \theta_{i}^{n}(z)-\sin \theta_{i+1}^{n}(z)}{g_{i}}\right|,
$$

where $i$ represents the $i$ th depth layer, $\Delta x_{i}, g_{i}$ and $\theta_{i}^{n}$ are respectively the horizontal propagation distance, the gradient of sound speed, and the grazing angle of the acoustic ray from the source to the $n$th HLA node at the $i$ th depth layer, and $c_{0}$ is the sound speed at the initial depth layer. When $i=0, \theta_{0}^{n}$ is the initial grazing angle 
Table 2: Virtual SSP generation algorithm for negative gradient "S" type shallow-water SSPs

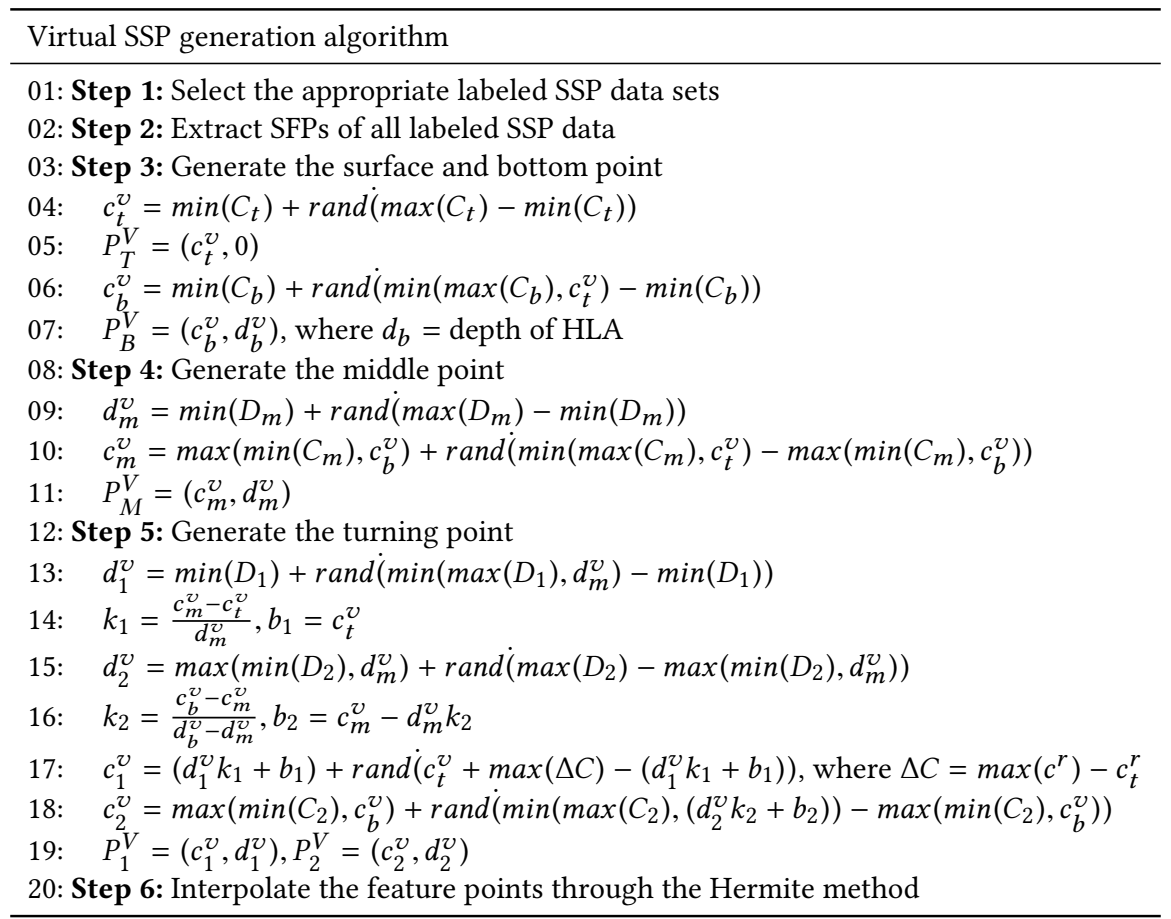

of the corresponding acoustic ray, and without signal reflection, the propagation time of the direct arrival signal to the $n$th HLA node is calculated by:

$$
t^{n}=\sum_{i=0}^{m} \Delta t_{i}=\sum_{i=0}^{m}\left|\frac{1}{g_{i}} \ln \frac{\tan \left(\frac{\pi}{4}+\frac{\theta_{i}^{n}(z)}{2}\right)}{\tan \left(\frac{\pi}{4}+\frac{\theta_{i+1}^{n}(z)}{2}\right)}\right|,
$$

where $\Delta t_{i}$ is the propagation time of the signal at the $i$ th layer. According to our sound field measuring method in section 2, the horizontal propagation distance between the $n$th pair of transceiver nodes is known as prior information. Thus, the initial grazing angle $\theta_{0}^{n}$ can be obtained by searching for the match of horizontal propagation distance at different initial grazing angles. Then the propagation time of the direct arrival signal at the $n$th HLA node can be calculated by substituting $\theta_{0}^{n}$ in $\operatorname{Eqn}(5)$.

\subsection{ANN-Based SSP Inversion}

The structure design of an ANN is important for improving its performance. The ANN adopted in this paper is a three-layer structure, as shown in Fig. 7. The input and hidden and output layer represent the propagation time of direct arrival signals, the implicit feature of the input and the corresponding SSP data, respectively. The number of input layer neurons depends on the number of HLA elements, and the number of output layer neurons is determined by the number of sampling points of the SSP. To avoid over-fitting and under-fitting problems, the number of hidden layer neurons should be well designed. However, to the best of our knowledge, there has been little accurate theory for determining this number yet, and it usually depends on the specific application. Therefore, inversion accuracy tests with different numbers of hidden layer

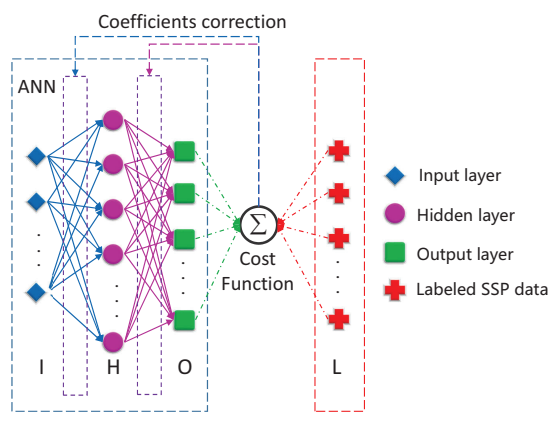

Figure 7: Structure of the three-layer ANN.

neurons are conducted to find an appropriate value in this paper, by which the influence of under-fitting and over-fitting issues caused by inappropriate hidden layer neuron numbers can be reduced.

To introduce nonlinear factors, the leaky rectified linear unit (LReLU) [8] is used between the input layer and the hidden layer. It expedites convergence of the training procedure and leads to better solutions than conventional sigmoid-like units. The LReLU function is expressed as:

$$
L(x)=\left\{\begin{array}{rr}
x & x>0 \\
a x & x \leq 0
\end{array}\right.
$$

where $a$ is a fixed constant between -1 and 0 .

To ensure good performance, the ANN needs to be well trained before application. During the training phase, most SSP data from the training SSP dataset are used for ANN training, and the weight 
coefficients of the ANN are updated by the back-propagation (BP) algorithm proposed in [11]. Then a small amount of data from the training SSP dataset is selected to verify whether the ANN has been trained well. The cost function to judge the performance of ANN is:

$$
C=\frac{1}{2} \sum_{p=1}^{P}\left(\bar{s}_{p}-s_{p}\right)^{2}
$$

where $P$ is the number of sample points of the training SSP, and $\bar{s}_{p}$ and $s_{p}$ stand for the sound speed value at the pth depth point of the inverted and training SSP data, respectively. The constant term is added for ease of calculation. When it comes to the working phase, the SSP inversion result is obtained by inputting the measured time information into the trained neural network. Because the training phase could be finished offline and only one forward propagation calculation is needed during the working phase, the computational time overhead of our method can be greatly reduced.

\section{SIMULATION RESULTS}

To avoid the over-fitting and under-fitting problems of ANN caused by inappropriate number of hidden layer neurons and training times, we study how these two parameters affect the accuracy performance of SSP inversion. Then the accuracy and efficiency performance of our approach are compared with traditional SSP inversion method based on MFP using EOF decomposition and PSO optimization algorithm (MFP-EOF-PSO).

The simulations are carried out in the MATLAB 2016 (a) simulatoe, and the empirical SSP data come from WOD13. Limited by the amount of empirical SSP data, we choose 21 sets of SSP data from 38-39 degrees north latitude, and 123.5-124.5 degrees west longitude along the eastern Pacific coast sampled in June with depth around 200 meters. The empirical SSP datasets are randomly divided into 3 parts that 15 sets used as empirical training data, 3 sets used as verification data, and 3 sets used as test data. The number of nodes in the HLA is assumed to be 9 in the simulation. The total training data are a combination of the empirical SSP data and the generated virtual SSP data according to the virtual SSP generation algorithm. To verify the feasibility of the virtual SSP generation algorithm, we generate 65 sets of virtual SSP data based on the 15 sets of empirical SSP data, and all SSP curves are given in Fig. 8 (a). The result indicates that the distribution of the virtual SSP data can be consistent with the empirical SSP data. Fig. 8 (b) shows the relationship between the root mean square error (RMSE) and the number of hidden-layer neurons for training 100 times. When the number of hidden-layer neurons is small or large, the RMSE of SSP inversion increases. This is due to the over-fitting and under-fitting problem, respectively, in the ANN training. Referring to the result, the number of hidden-layer neurons is determined to be 80 in our experiment. Fig. 8 (c) shows the convergence performance of our method with different training times. Considering all the 3 sets of verification data, the system stabilizes and converges when the times of training exceed 100 repetitions. To ensure stable convergence of the neural network, the number of training times in the subsequent simulation is set to be 200 repetitions.

The EOF decomposition and heuristic optimization algorithms are widely used in the MFP-based SSP inversion method. In this paper, the PSO optimization algorithm is employed as a representation of heuristic optimization algorithms to be compared with

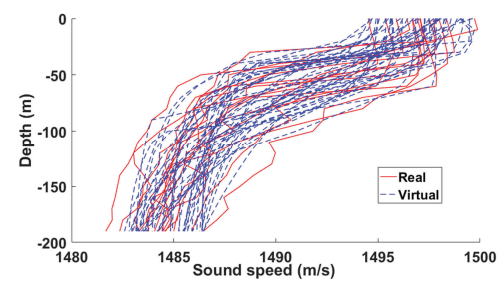

(a) Training data set.
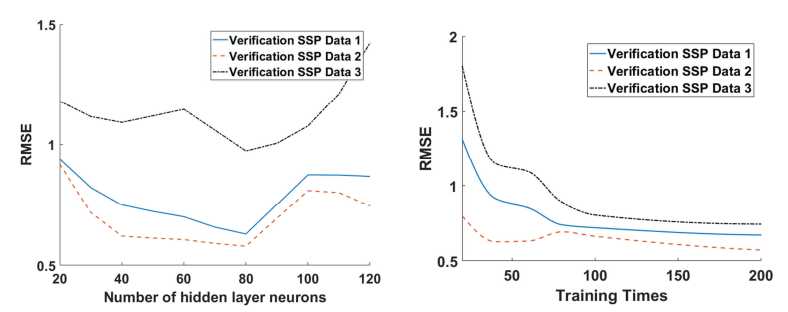

(b) RMSE of SSP inversion as a function (c) RMSE of SSP inversion as a function of the number of hidden layer neurons. of training times.

Figure 8: The impact of the number of hidden layer neurons and ANN training times on RMSE.

Table 3: Setting of simulation parameters

\begin{tabular}{cc}
\hline Item & Value \\
\hline Number of input-layer neurons & 9 \\
Number of hidden-layer neurons & 80 \\
Number of output-layer neurons & 20 \\
Learning rate & 0.0001 \\
PSO particles & 20 \\
PSO iteration times & 50 \\
Input time noise & $-5 \mu \mathrm{s}$ \\
Number of training SSP data & 200 \\
Number of verification SSP data & 3 \\
Number of test SSP data & 3 \\
Sample depth interval of SSP & $10 \mathrm{~m}$ \\
\hline
\end{tabular}

our approach. The key parameters of our method and the PSO optimization algorithm are listed in Table 3.

The time overhead result at different phases on the 3 sets of test data is presented in Fig. 9. The time overhead at the working phase of the ANN is less than that of the MFP-EOF-PSO, which is equivalent to one time iteration of a single particle. In fact, time is mainly consumed at the working phase due to the massive matching search calculations for MFP-EOF-PSO. For an ANN, the computational overhead is transferred from the working phase to the training phase. In this way, the energy of underwater nodes can be saved because the training work can be finished offline before the task. What's more, reducing computation during the working phase makes it better to monitor environmental parameters in real time.

To test the accuracy performance of our approach, the RMSE result of ANN is compared with that of MFP-EOF-PSO in Fig. 10 


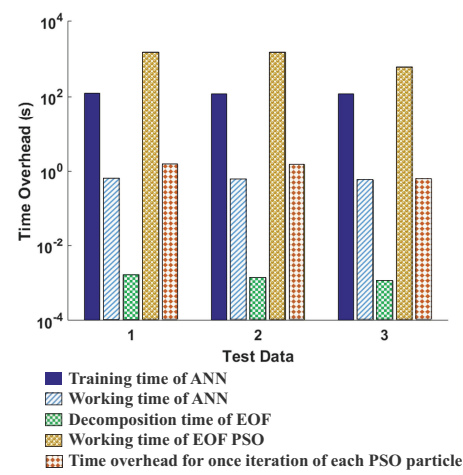

Figure 9: Comparison of time overhead at different phases between ANN and EOF PSO.
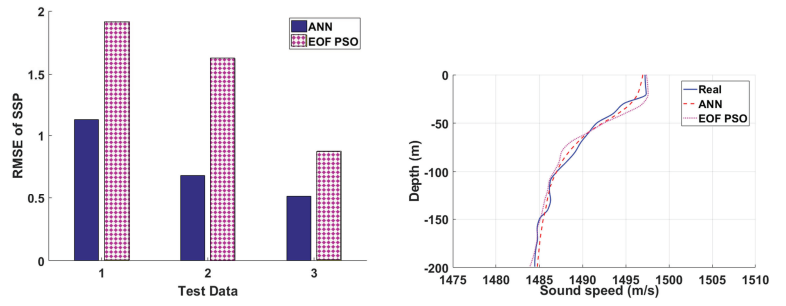

(a) Comparison of inversion accuracy (b) SSP inversion result fot test data 1 . between ANN and EOF PSO.

Figure 10: The accuracy of SSP inversion results.

(a), and the result of test data 1 is typically given as an example in Fig. 10 (b). Simulation results prove that our approach can achieve a good inversion accuracy for SSP inversion in shallow water.

\section{CONCLUSION}

In this paper, we propose a joint ANN and ray theory shallow-water SSP inversion method, which uses the propagation time of direct arrival signals measured by the two-way interactive communication between an AUV and an HLA. In this manner, the influence of any boundary parameter mismatch can be reduced. To make the ANN better learn the SSP distribution in a target region, we propose an empirical SSP data selection strategy. To solve the under-fitting problem caused by insufficient training data, we propose a virtual SSP data generation algorithm based on the SFP extraction results of the empirical SSP data, and the virtual SSP data is combined with the empirical SSP data for ANN training. Simulation results have verified the feasibility and effectiveness of our approach that the time overhead at the working phase can be reduced while ensuring accuracy.

In our future work, we will further explore the anti-noise performance of our approach, extend it to deeper ocean areas, and verify our approach in an ocean trial.

\section{ACKNOWLEDGMENTS}

The authors would like to thank the National Oceanic and Atmospheric Administration for providing the CTD database. The authors would also like to thank the anonymous referees for their valuable comments and helpful suggestions. The work is supported by the National Natural Science Foundation of China under Grant No. 2014AA09A512.

\section{REFERENCES}

[1] J. V. Candy and E. J. Sullivan. 1986. Sound Velocity Profile Estimation: A System Theoretic Approach. IEEE fournal of Oceanic Engineering 18, 3 (July 1986), 240 252. https://doi.org/10.1109/JOE.1993.236362

[2] D. F. Dinn, B. D. Loncarevic, and G. Costello. 1995. The Effect of Sound Velocity Errors on Multi-beam Sonar Depth Accuracy. In 'Challenges of Our Changing Global Environment' Conference Proceedings. OCEANS '95 MTS/IEEE, Vol. 2. IEEE, San Diego, California, USA, 1001-1010. https://doi.org/10.1109/OCEANS.1995. 528559

[3] J. J. Hopfield. 1988. Artificial Neural Networks. IEEE Circuits and Devices Magazine 4, 5 (1988), 3-10. https://doi.org/10.1109/101.8118

[4] F. B. Jensen, W. A. Kuperman, M. B. Porter, and H. Schmidt. 2011. Computational Ocean Acoustics (2nd. ed.). Springer New York, New York, NY. 117-121 pages.

[5] F. H. Li and R. H. Zhang. 2010. Inversion for Sound Speed Profile by Using a Bottom Mounted Horizontal Line Array in Shallow Water. Chinese Physics Letters 27, 8 (Aug 2010), 084303. https://doi.org/10.1088/0256-307X/27/8/084303

[6] Z. L. Li, H. Li, R. H. Zhang, F. H. Li, Y. X. Yu, and L. Peng. 2015. Sound Speed Profile Inversion Using A Horizontal Line Array in Shallow Water. Science China Physics, Mechanics \& Astronomy 58, 1 (Jan 2015), 1-7. https://doi.org/10.1007/ s11433-014-5526-x

[7] J. Liu, Z. Wang, J. Cui, S. Zhou, and B. Yang. 2016. A Joint Time Synchronization and Localization Design for Mobile Underwater Sensor Networks. IEEE Transactions on Mobile Computing 15, 3 (March 2016), 530-543. https: //doi.org/10.1109/TMC.2015.2410777

[8] A. L. Maas, A. Y. Hannun, and A. Y. Ng. 2013. Rectifier Nonlinearities Improve Neural Network Acoustic Models. In ICML'13: Proceedings of the 30th International Conference on International Conference on Machine Learning, Sanjoy Dasgupta and David McAllester (Eds.), Vol. 28. JMLR.org, Atlanta, Georgia, USA. http: //robotics.stanford.edu/ amaas/papers/relu_hybrid_icml2013_final.pdf

[9] W. Munk and C. Wunsch. 1979. Ocean Acoustic Tomography: A Scheme for Large Scale Monitoring. Deep Sea Research Part A. Oceanographic Research Papers 26, 2 (1979), 123 - 161. https://doi.org/10.1016/0198-0149(79)90073-6

[10] W. Munk and C. Wunsch. 1983. Ocean Acoustic Tomography: Rays and Modes. Reviews of Geophysics 21, 4 (May 1983), 777-793. https://doi.org/10.1029/ RG021i004p00777

[11] D. E. Rumelhart, G. E. Hinton, and R. J. Williams. 1986. Learning Representations by Back-propagating Errors. Nature Publishing Group 323, 6088 (1986), 533. https://doi.org/10.1038/323533a0

[12] E. C. Shang. 1989. Ocean Acoustic Tomography Based on Adiabatic Mode Theory. Acoustical Society of America fournal 85, 4 (Apr 1989), 1531-1537. https://doi. org/10.1121/1.397355

[13] W. C. Sun, J. Y. Bao, S. H. Jin, F. M. Xiao, and Y. Cui. 2016. Inversion of Sound Velocity Profiles by Correcting the Terrain Distortion. Geomatics and Information Science of Wuhan University 41, 3 (2016), 349-355. https://doi.org/10.13203/j. whugis 20140142

[14] J. F. Tang and S. E. Yang. 2006. Sound Speed Profile in Ocean Inverted by Using Travel Time. Fournal of Harbin Engineering University 27, 5 (11 2006), 733-736+756. https://doi.org/10.3969/j.issn.1006-7043.2006.05.022

[15] A. Tolstoy, O. Diachok, and L. N. Frazer. 1991. Acoustic Tomography Via Matched Field Processing. The fournal of the Acoustical Society of America 89, 3 (1991), 1119-1127. https://doi.org/10.1121/1.400647

[16] Q. Y. Wu and W. Xu. 2017. Matched Field Source Localization As A Multiple Hypothesis Tracking Problem. In Proceedings of the International Conference on Underwater Networks \& Systems, Vol. 7. ACM, Halifax, NS, Canada, 25:1-25:2. https://doi.org/10.1145/3148675.3148723

[17] W. Zhang. 2013. Inversion of Sound Speed Profile in Three-dimensional Shallow Water. Phdthesis. Harbin Engineering University, Harbin, China.

[18] W. Zhang, S. E. Yang, Y. W. Huang, and L. Li. 2012. Inversion of Sound Speed Profile in Shallow Water with Irregular Seabed. AIP Conference Proceedings 1495, 1 (2012), 392-399. https://doi.org/10.1063/1.4765934

[19] Z. M. Zhang. 2005. The Study for Sound Speed Inversion in Shallow Water on Application of Genetic and Simulated Annealing Algorithms. Master's thesis. Harbin Engineering University, Harbin, China. https://doi.org/10.7666/d.y780567

[20] G. Y. Zheng and Y. W. Huang. 2017. Improved Perturbation Method for Sound Speed Profile Inversion. Journal of Harbin Engineering University 38, 3 (2017), 371-377. https://doi.org/10.11990/jheu.201603075 